\title{
Modelling Long-term Bridge Deterioration at Structural Member Level Using Artificial Intelligence Techniques
}

\author{
Jaeho Lee ${ }^{1, a}$, Hong Guan ${ }^{1, b}$, Yew-Chaye Loo ${ }^{2, c}$, Michael Blumenstein ${ }^{2, d}$ and \\ Wang Xin-ping ${ }^{3, \mathrm{e}}$
} ${ }^{1}$ Centre for Infrastructure and Engineering Management (CIEM), Gold Coast Campus, Griffith
University, QLD 4222, Australia

${ }^{2}$ Science, Environment, Engineering \& Technology, Griffith University, QLD 4222, Australia

${ }^{3}$ School of Civil Engineering, Shandong Jianzhu University, Jinan 250014, China

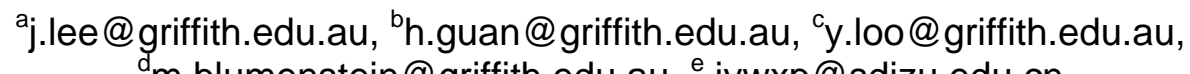
dm.blumenstein@griffith.edu.au, ${ }^{e}$ jywxp@sdjzu.edu.cn

Keywords: Bridge Asset Management, Bridge Management System (BMS), Bridge Deterioration, maintenance, repair and rehabilitation (MR\&R), Artificial Intelligence (AI), Backward Prediction Model (BPM)

\begin{abstract}
Efficient use of public funds for structural integrity of bridge networks requires an effective bridge asset management technology. To achieve this, a reliable deterioration model is essential in any Bridge Management System (BMS). The deterioration rate is calculated based on historical condition ratings obtained from the structural element-level bridge inspections. Although most bridge authorities have previously conducted inspection and maintenance tasks, these past inspection records are incompatible with what are required by a typical BMS as input. Such incompatibility is a major cause for the deficiency of the current BMS outcomes. Artificial Intelligence (AI)-based bridge deterioration model has recently been developed to minimise uncertainties in predicting deterioration of structural bridge members (e.g. beams, piers etc). This model contains two components: (1) using Neural Network-based Backward Prediction Model (BPM) to generate unavailable historical condition ratings; and (2) using Time Delay Neural Network (TDNN) to perform long-term performance prediction of bridge structural members. However new problems have emerged in the process of TDNN prediction. This is because the BPM-generated condition ratings are used together with the actual condition ratings. The incompatibility between the two sets of data produces unreliable prediction outcomes during the TDNN process. This research is thus to develop a new process based on the existing method, thereby overcoming the abovementioned problems. To achieve this, the actual overall condition ratings are replaced by the BPM forward predicted condition ratings. Consequently, the outcome of this study can improve accuracy of long-term bridge deterioration prediction.
\end{abstract}

\section{Introduction}

Most state and local bridge authorities have become aware of the urgency to develop management technology to ensure safe performance of bridge networks and to facilitate continuous economic growth. Basically, asset management methodologies incorporate economic assessments leading to cost-effective long-term decisions concerning maintenance, repair and rehabilitation (MR\&R). It is particularly important to plan minimum-cost MR\&R activities with funding allocations based on reliable Bridge Management System (BMS) outcomes. BMSs are already a common tool for many bridge authorities to extend the life of bridge networks. However, the reliability of current BMS outcomes is doubtful.

Bridge deterioration is a stochastic process and the causes of deteriorations are due to diverse explanatory variables including "non-bridge factors" such as traffic intensity, environmental factors, climates etc [1,2]. Collection of such variables is not included in current bridge inspection routines. 
Because of this and with only limited bridge condition rating records, it is very difficult to use typical stochastic-based deterioration models to accurately predict future condition ratings. This limitation has been recognised internationally and it is a challenging problem that has not yet been addressed [3-6]. A deterioration model is the most critical component of a BMS. The deterioration rate is calculated based on historical condition ratings obtained from the structural element-level bridge inspections [7]. Although most bridge authorities have previously conducted inspection, these past inspection records are incompatible with what are required by a typical BMS as input. Such incompatibility is a major cause for the deficiency of the current BMS outcomes. Because of this sheer lack of usable inspection records i.e. insufficient historical condition ratings, the outcomes of all current BMSs which incorporate a deterioration model of one type or another are inadequate and unreliable [8]. For example, the China Bridge Management System (CBMS) has shown, in its early stage of operation that the reliability of BMS outcomes remains questionable [9]. Any BMS should provide effective evaluation and prediction of future bridge performance to aid optimum MR\&R expenditure decisions. Due to the lack of historical inspection data and the weakness of using the weighted overall bridge condition rating method, subjective and qualitative analyses are therefore required for rating, sorting, and planning of future bridge needs. Moreover, the essential deterioration curves, statistic assessment and optimisation techniques are not included in the current CBMS. In other words, the CBMS currently functions more like a Bridge Information System (BIS). This is not the only issue for China. Due to the lack of historical condition rating information, many of bridge authorities worldwide have similar problems in using BMS for accurate and reliable prediction of long-term bridge performance and budget planning.

Further limitation with the current deterioration model is related to the Overall Condition Rating (OCR) methodology used in most existing bridge management technologies. At present, the condition rating information is collected via a quantitative bridge inspection procedure; it is then converted into OCR in a subjective manner. The conditions of bridge elements collected using the element-level bridge inspection process, are expressed quantitatively via the conventional "grading" system, i.e. the health index or the four condition states (CSs 1 to 4). The overall condition of one or more element types of a bridge is calculated with the aid of a weighted average condition state (CS) numbering system. Thus the OCR is incapable of capturing the condition status of individual structural members (i.e. individual beams, piers etc), be it at CS1 (i.e. condition as new or "good"), CS2 ("fair"), CS3 ("poor") or the perilous CS4 ("very poor"). This is a key drawback because bridge collapse usually occurs as a result of the failure of single member(s). In view of this, each of the four CSs for individual members needs to be evaluated in order to reduce the risks of total bridge failure. A further drawback with this stepwise "grading" system is that there are only four CSs with graduation of $1 / 4$ or $25 \%$. Such a step is too large to be used effectively in deterioration modelling. For example, for a CS2 rating the numerical weighting is $62.5 \%$ which is the average of 75 and 50\%, whereas in reality the condition of the member can be anywhere between "as new" and "defective". This indeterminacy seriously increases the degree of uncertainty with time in predicting long-term bridge element performance. Note also that it is too expensive to change the current inspection method, which has been used for many years and already produced massive amounts of historical condition rating records. Any change to the inspection method will also create data-incompatibility issues.

In order to address these problems, a study has been conducted recently in an attempt to generate unavailable bridge condition ratings [8]. This is referred to as the Artificial Intelligence (AI)-based Backward Prediction Model (BPM), which provides unknown historical bridge deterioration patterns to assist in predicting reliable long-term bridge deteriorations. The mechanism of the BPM is described schematically in Figure 1. It illustrates the main function of the ANN technique in establishing the correlation between the existing condition rating datasets and the corresponding years' non-bridge factors, such as traffic volume and climatic conditions. The non-bridge factors directly and indirectly affect the variation of the bridge conditions and thereby the deterioration rate. The relationships established using neural networks are then applied to the missing years' nonbridge factors to generate the missing bridge condition ratings. 


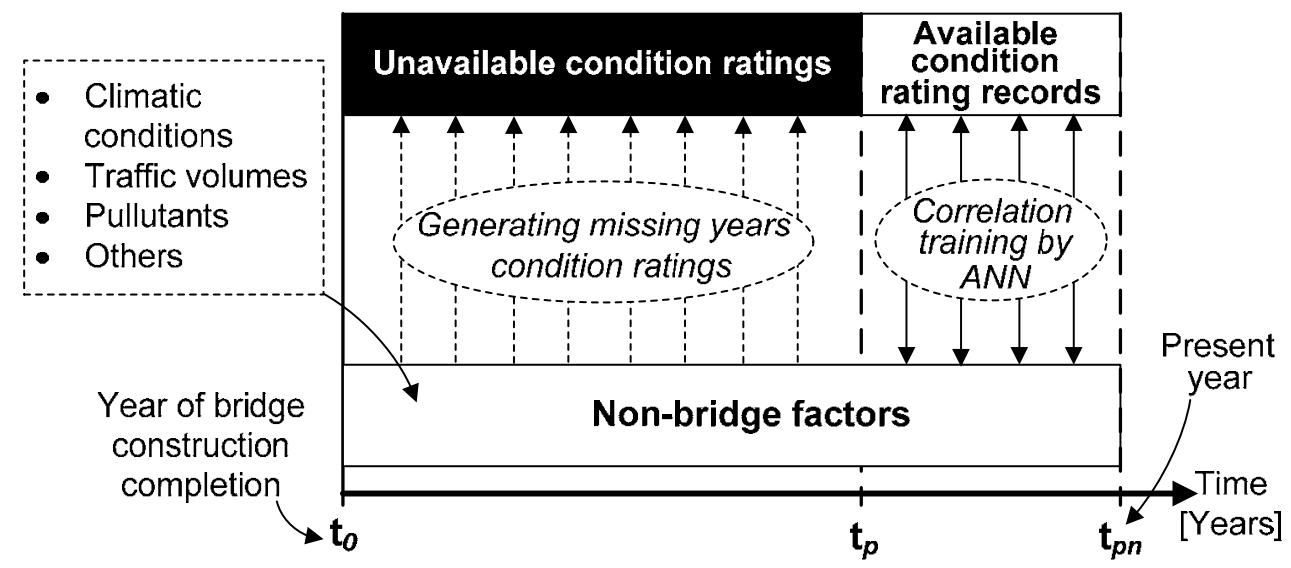

Figure 1: Structure of Backward Prediction Model (BPM)

Comparisons with typical bridge deterioration models have also been conducted demonstrating that the prediction errors decrease when the amount of input data increases [9]. This provides further confirmation for the effectiveness of the BPM. In addition to the above, a study has also been conducted using AI-based bridge deterioration modelling technique incorporating the BPM to improve accuracy of long-term prediction [8,10]. However, a fundamental shortcoming is discovered in predicting long-term performance of bridge elements due to the use of weighted overall condition rating method. This causes mismatch with the BPM-generated condition ratings which in turn leads to inaccurate long-term predictions. This paper is thus aimed at developing a new analysis process to the existing BPM-based deterioration method thereby minimising the abovementioned problems.

\section{Outline of the Study}

The BPM methodology in conjunction with the Time-Delay Neural Networks (TDNNs) technique has been employed to overcome the fundamental shortcomings mentioned above [10]. A two-stage procedure for the proposed AI-based deterioration model is presented in Figure 2. Also detailed in the figure are indications of the time periods: available condition ratings ( $t_{p}$ to $\left.t_{p n}\right)$; BPM inputs $\left(t_{0}\right.$, $t_{p}$ to $\left.t_{p n}\right)$; BPM outputs ( $t_{1}$ to $\left.t_{p-1}\right)$; BPM and TDNN validation using BPM outputs ( $t_{1}$ to $\left.t_{p-1}\right)$; and TDNN long-term prediction ( $t_{\mathrm{f} 1}$ to $t_{\mathrm{fn}}$ ) using condition ratings ( $t_{0}$ to $\left.t_{p n}\right)$. Note that $t_{0}$ refers to the year of construction completion and its condition rating is assumed to be excellent in this study.

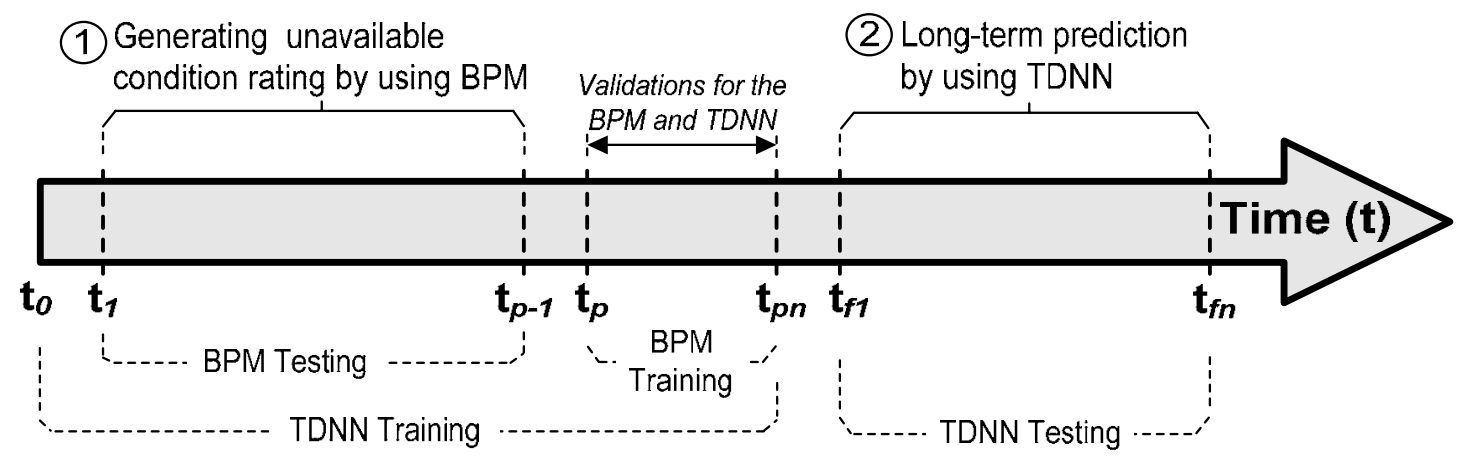

Fig. 2. The proposed AI-based bridge deterioration model

In Stage 1 , to generate unavailable historical condition rating ( $t_{1}$ to $t_{\mathrm{p}-1}$ ), an Artificial Neural Network (ANN)-based BPM is used to establish a correlation between the existing condition rating datasets $\left(t_{p}\right.$ to $\left.t_{p n}\right)$ and the corresponding years' non-bridge factors. The correlations established are then applied to the non-bridge factors ( $t_{1}$ to $t_{p-1}$ ) to generate (backward predict) missing bridge condition ratings ( $t_{1}$ to $\left.t_{p-1}\right)$. The non-bridge factors, in combination with the ANN technique, can 
thereby reproduce the historical deterioration trends. The BPM-generated condition ratings for each year contains 66 spectrum rating numbers per CS, which is a combination of learning rates (lr: 0.00.5 @0.1 increment resulting 6 lr) and momentum coefficients (mc: 0.0-1.0 @ 0.1 increment resulting $11 \mathrm{mc}$ ) in the Neural Network configurations. The number 66 also relates to the total quantity of a given bridge element. The cross-validation is utilised to confirm the reliability of the $\mathrm{BPM}$ results. The BPM produces forward prediction ( $\left.\mathrm{t}_{\mathrm{p}} \mathrm{to}_{\mathrm{pn}}\right)$ using BPM-generated outcomes ( $\mathrm{t}_{1}$ to $\left.t_{\mathrm{p}-1}\right)$ only as input values. The results of the forward predictions are then compared with the actual BMS condition ratings $\left(t_{p}\right.$ to $\left.t_{p n}\right)$. Once validated, the BPM backward prediction $\left(t_{1}\right.$ to $\left.t_{p-1}\right)$ together with its forward prediction ( $t_{p}$ to $t_{p n}$ ) are eligible to be used as input values for long-term predictions to be carried out in Stage 2.

There is a problem however, when the BPM backward prediction ( $t_{1}$ to $\left.t_{p-1}\right)$ and its forward prediction ( $t_{p}$ to $t_{p n}$ ) are used together as TDNN input, some of the BPM predicted condition ratings are found to be slightly higher than those of the preceding years. Bridge deterioration is a stochastic process and may progress continuously, gradually and slowly [1]. Thus, the condition rating should not be improved if no MR\&R, i.e. preservation, is undertaken. In other words, this study considers "Do-nothing" - no maintenance effects in long-term prediction. The higher predicted condition ratings cause confusion during the TDNN training session which in turn can yield less-accurate predictions during TDNN testing. To minimise this problem, all BPM-generated condition ratings are required to undergo a filtering process for further improvement of long-term prediction quality. This process is a new addition to the originally developed AI-based deterioration model. The filtering process follows a simple criterion, i.e. Condition Rating (year $n-1$ ) $\geq$ Condition Rating (year $n$ ).

In Stage 2, the outcomes of filtered data ( $t_{1}$ to $\left.t_{\mathrm{pn}}\right)$ from Stage 1 and assumed condition rating of the initial year $\left(\mathrm{t}_{0}\right)$ are used as TDNN input to predict long-term bridge element performance. The input data is converted to time sequences for time series prediction in the TDNN. The TDNN provides only one-step ahead prediction at a time (one cycle), i.e. 1-year interval in the actual time domain. The result of the first one-step-ahead prediction is added onto the original TDNN input $\left(\mathrm{t}_{0}\right.$ to $\left.t_{\mathrm{pn}}\right)$. This indicates that the number of inputs to the TDNN increases in the second cycle of the one-step-ahead prediction. Iterations of the above-described process are required until prediction up to year $t_{f n}$ is completed. The number of yearly prediction by TDNN is also 66, which is in an identical form as the BPM outcomes obtained in Stage 1. Cross-validation is also required to evaluate prediction accuracy of the TDNN outcomes. This is done by comparing the results of TDNN predictions ( $t_{p}$ to $\left.t_{p n}\right)$ to the known or "actual” BMS condition ratings ( $t_{p}$ to $\left.t_{p n}\right)$. All TDNN outcomes are considered acceptable if the comparisons are within the same Condition State of the original inspection records. It should be noted that the final results of the TDNN, upon calibration, are also in the same form as the bridge element-level inspection.

\section{Improvement of Existing AI-based Model}

This study is conducted using the sample bridge condition rating datasets (i.e. Reinforced Concrete Pier Cap) provided by the Maryland Department of Transport (DoT), USA. Figure 3 describes the timeframe of input and output for the proposed deterioration model. Note that only three sets of actual condition rating records (from years 1996 to 2000 with a 2-year increment) are available for both BPM and TDNN. The timeframe shows: (a) available condition ratings; (b) BPM inputs; (c) BPM-generated historical condition ratings; (d) BPM validation inputs; (e) BPM validation outputs; (f) TDNN validation inputs; (g) TDNN validation outputs; (h) TDNN inputs; and (i) TDNN outputs for long-term prediction of bridge elements. Detailed in the following sub-sections are the study outcomes for Stages 1 and 2. 


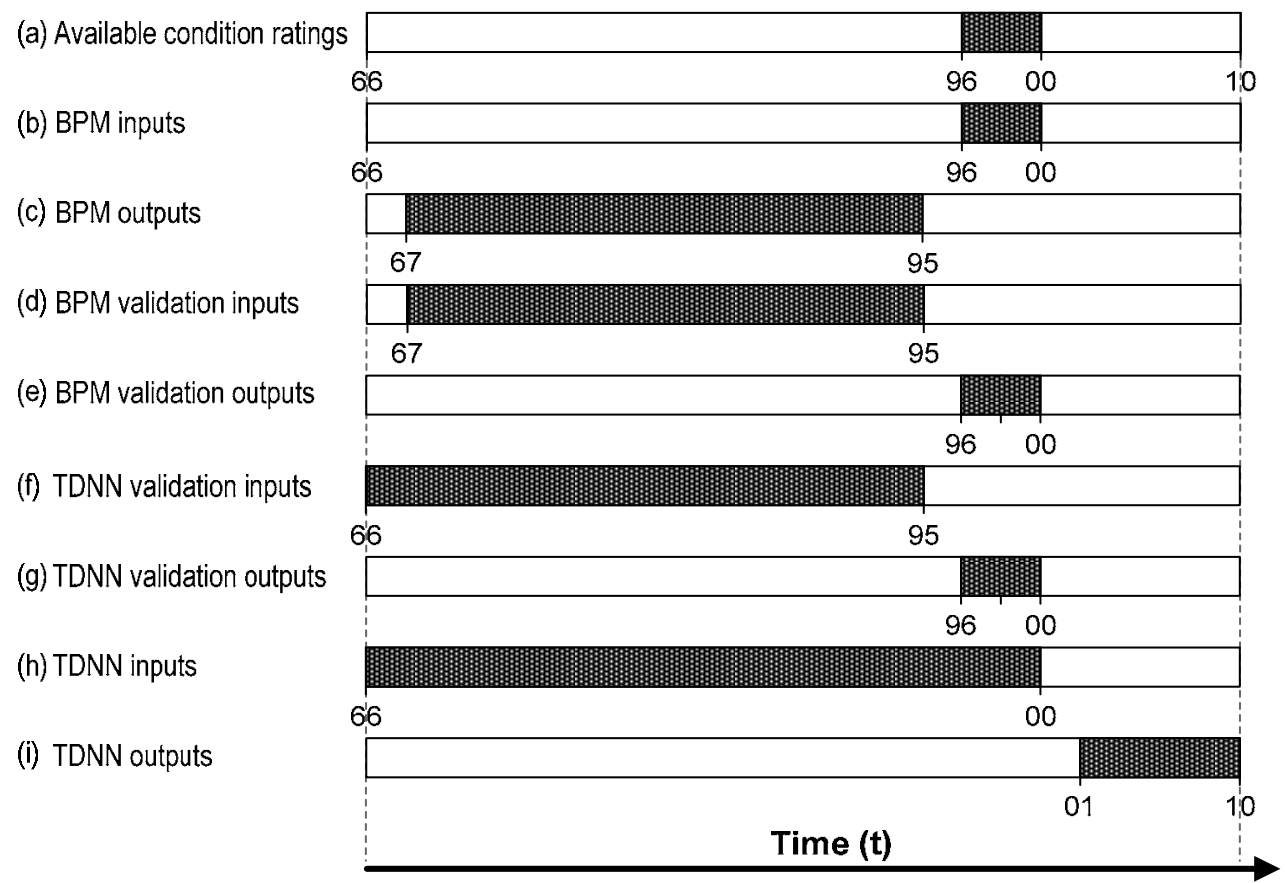

Fig. 3. Timeframe of testing the proposed deterioration model

\section{STAGE 1: Backward Prediction Model (BPM)}

BMS condition rating records obtained from the Maryland DoT is related to a superstructure element - Reinforced Concrete Pier Cap (Element \#234 on Bridge \#0301xxxx1). The actual BMS condition rating inputs are collected to periodically update the BMS database. The Condition States (CSs) of BMS condition ratings is scaled between CS1 (excellent) and 5 (fail) for the bridge element. The original condition rating information is detailed in Table 1.

Table 1. Raw data of actual condition ratings

\begin{tabular}{|c|c|c|c|c|c|c|}
\hline \multirow{2}{*}{ Year of inspection } & \multicolumn{7}{|c|}{ Quantity (EA, \%) } \\
\cline { 2 - 7 } & Total & CS1 & CS2 & CS3 & CS4 & CS5 \\
\hline 1996 & $350(100)$ & $280(80)$ & $50(14)$ & $20(6)$ & 0 & 0 \\
\hline 1998 & $350(100)$ & $280(80)$ & $50(14)$ & $20(6)$ & 0 & 0 \\
\hline 2000 & $350(100)$ & $280(80)$ & $50(14)$ & $20(6)$ & 0 & 0 \\
\hline Average (\%) & 100 & 80 & 14 & 6 & 0 & 0 \\
\hline
\end{tabular}

Generating historical condition ratings: The average quantity of each CS between 1996 and 2000 is about $80 \%, 14 \%$ and $6 \%$ of the total element quantities in CS1, CS2 and CS3, respectively. Figure 4 demonstrates that the BPM generates historical condition ratings from 1967 to 1995 (i.e. 29 years) in three different proportions of the element quantity using the existing condition ratings (1996 to 2000, i.e. 5 years) together with the assumed condition rating (CS1-Excellent) at initial year ( $t_{0}$, i.e. 1966). 


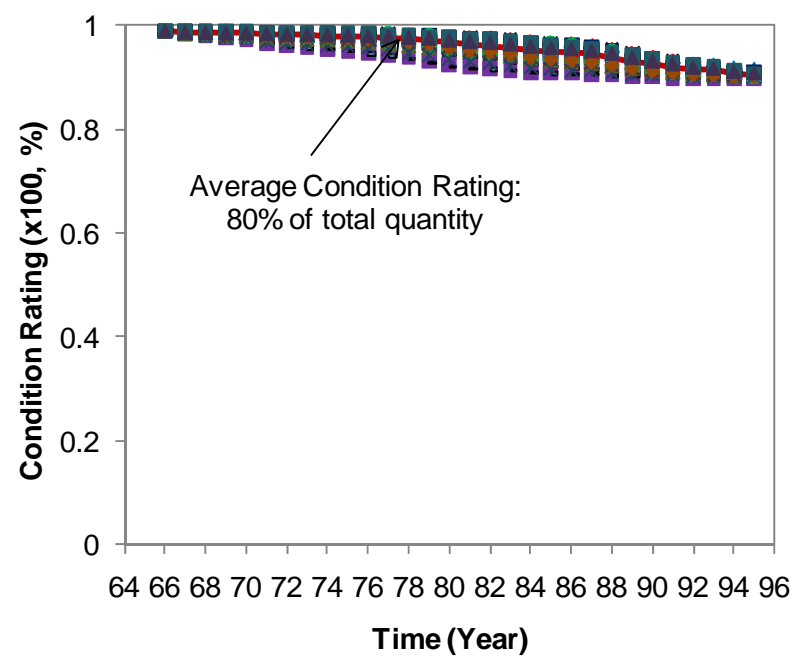

(a) $80 \%$ of total element quantity

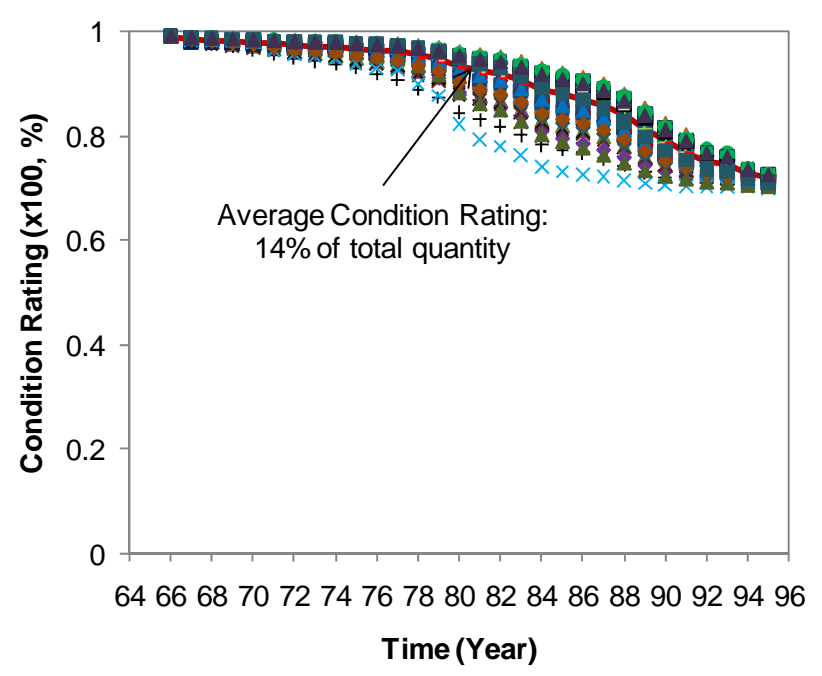

(b) $14 \%$ of total element quantity

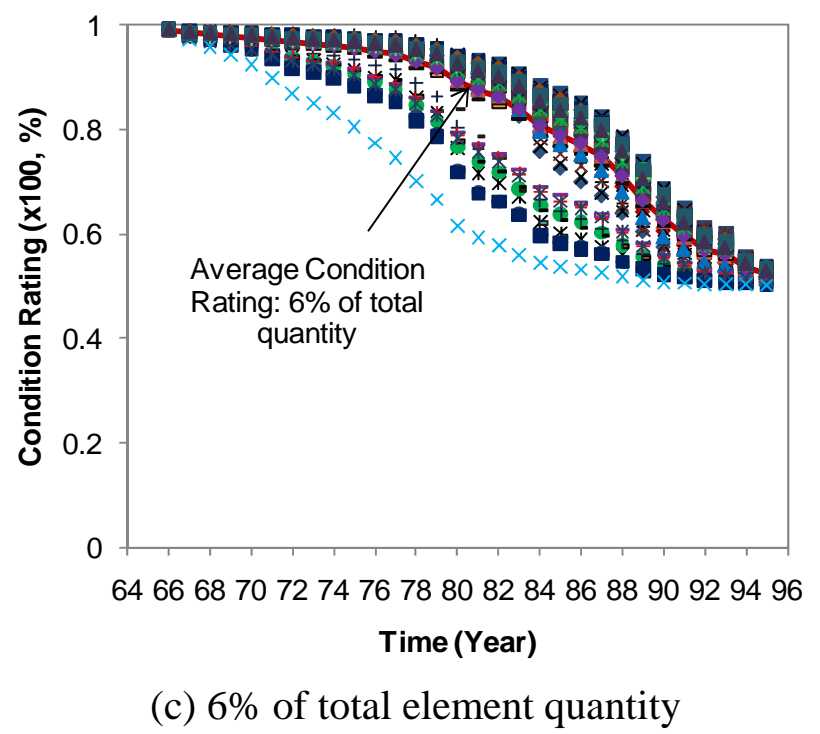

Fig. 4. BPM Results (Note that the number of outcomes in each year is 66 which is the combined number of learning rate (lr: 0.0-0.5) and momentum coefficient (mc: 0.0-1.0) in the neural network configuration)

Cross validation of BPM-generated condition ratings: To validate the BPM outcomes, only generated historical condition ratings (years 1967 to 1995) are used as input for forward-prediction of the present years (years 1996-2000). Figure 5 shows prediction results (years 1996 to 2000) for three different proportions of the element quantity, i.e. $80 \%, 16 \%$ and $4 \%$. Note that only 3 years' condition ratings (years 1996, 1998 and 2000) can be used to conduct cross-validation. In this study, the maximum prediction error allowance is defined as $\pm 10 \%$. This is half magnitude of a Condition State for a 5 CS-scale. In Figure 5 (c), the predicted minimum condition rating in year 2000 is $35.8 \%$. This value, compared to the lower bound of CS3 (40\% to 60\%), results in a $4.2 \%$ prediction error (CS3min - Predicted condition rating $=40 \%$ to $35.8 \%$ ). This is within the error allowance. Therefore, cross-validation of BPM-generated condition rating is considered satisfactory.

Data Preprocessing: For realistic forecast of the deterioration rates, the BPM-generated condition ratings require data preprocessing (or filtering) to remove meaningless condition ratings that causes poor training outcomes for long-term prediction using TDNN. Some of the BPM predicted condition ratings are found to be slightly higher than those of the preceding years. This does not match with the absence of MR\&R activities. The total number of yearly prediction by TDNN is 198, as a result of 66 cases for each of the three proportions $(80 \%, 14 \%, 6 \%$ of total element quantity). It 
is observed that 88 cases out of the total 198 cases have their condition ratings predicted unrealistically higher. The maximum errors are respectively $0.61 \%, 3.26 \%, 1.14 \%$ in $80 \%, 14 \%$, $6 \%$ of the total element quantity. The corresponding average maximum errors are $0.41 \%, 1.49 \%$, $0.69 \%$. Through a data filtering process, the BPM-generated condition ratings satisfying the following criteria, Condition Rating(year $\underline{\mathrm{n}-1}$ ) $\geq$ Condition Rating(year $\underline{\mathrm{n}}$ ), should be removed and replaced by the identical condition rating of the preceding year.

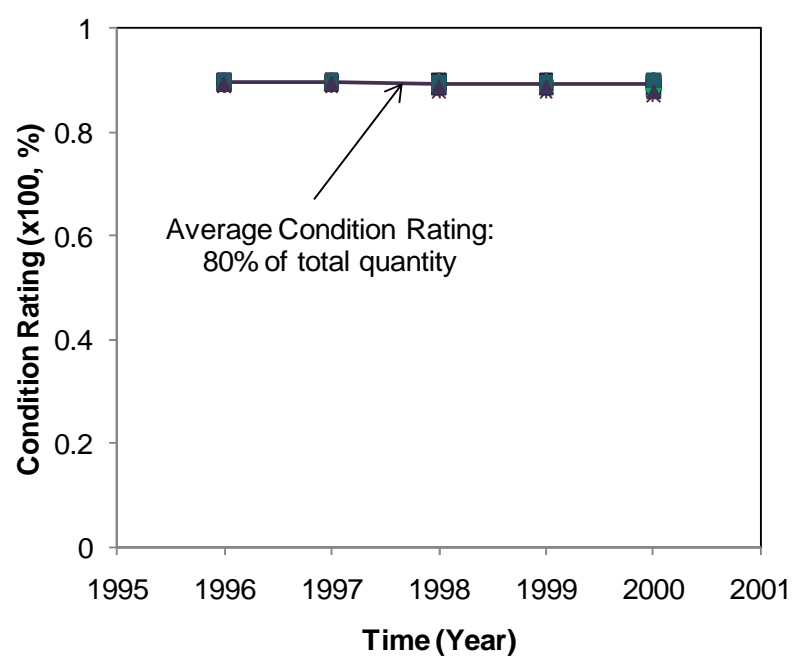

(a) $80 \%$ of total element quantity

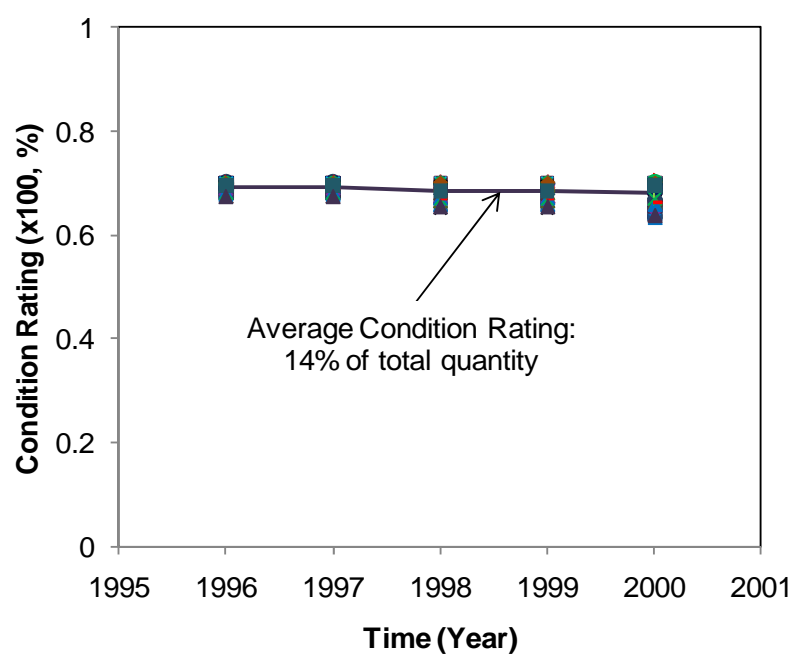

(b) $14 \%$ of total element quantity

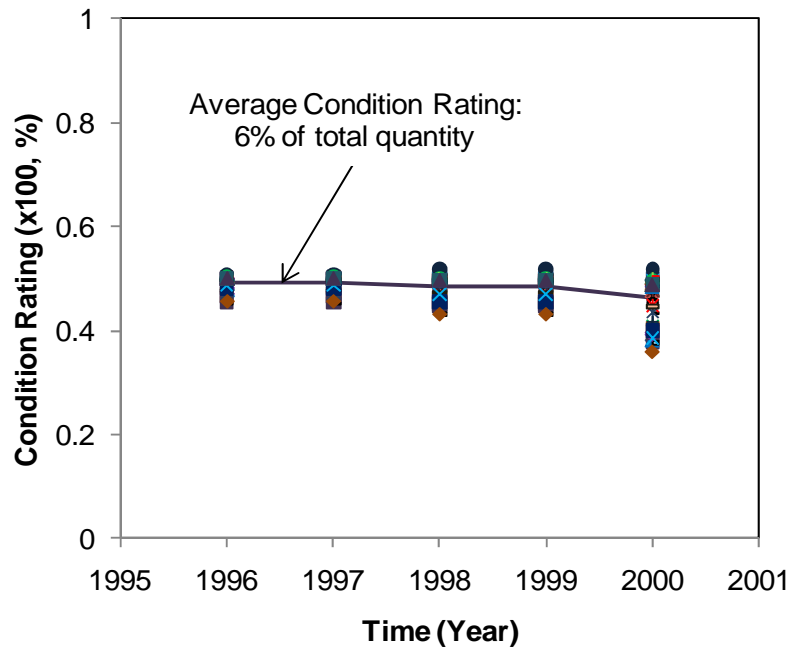

(c) $6 \%$ of total element quantity

Fig. 5. BPM results for Cross-validation (1996, 1998, and 2000)

\section{STAGE 2: Time-Delay Neural Networks (TDNNs)}

Before performing long-term prediction for years 2001-2010, the TDNN outputs are required to be validated. A short-term prediction for years 1996 to 2000 is considered for cross-validation to measure prediction accuracy of the proposed deterioration model.

A short-term prediction for cross-validation: The BPM-generated historical condition ratings (years 1967 to 1995) together with the assumed condition rating at the year of construction completion (year 1966) are used as TDNN inputs for the short-term prediction (for years 1996 to 2000). One-step-ahead TDNN prediction is conducted up until year 2000. The outcomes (years 1996 to 2000) of TDNN are subsequently compared with the actual condition rating records (years 1996, 1998, 2000) for further confirmation of BPM-generated data. The TDNN short-term prediction, as cross-validation, is presented in Figure 6. It is found in Figure 6 (c) that the minimum 
condition rating predicted for CS3 in year 2000 is 38.75\%. When compared to the lower bound of CS3 (40\% to 60\%), a $1.25 \%$ (CS3min - predicted condition rating $=40 \%$ to $38.75 \%$ ) prediction error is produced which is within the error allowance $( \pm 10 \%)$. Therefore, cross-validation for TDNN short-term prediction using BPM-generated condition ratings is also considered satisfactory.

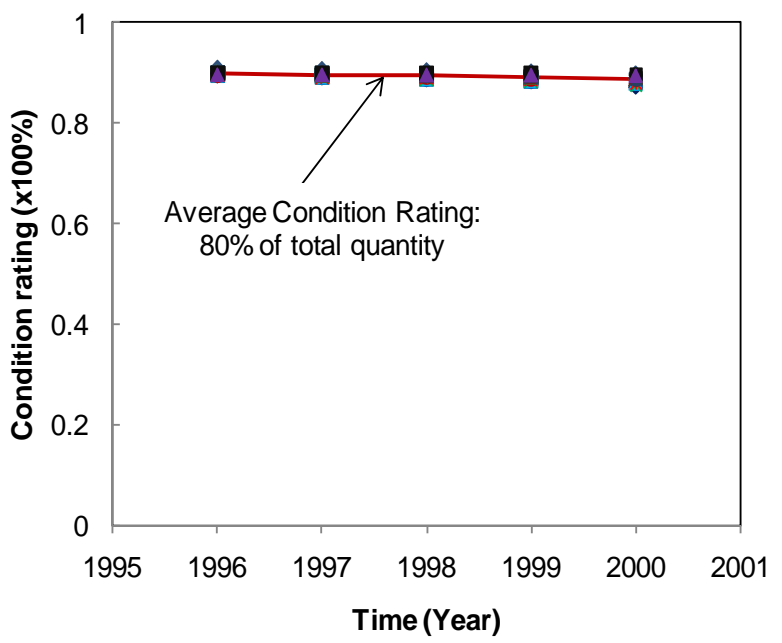

(a) $80 \%$ of total element quantity

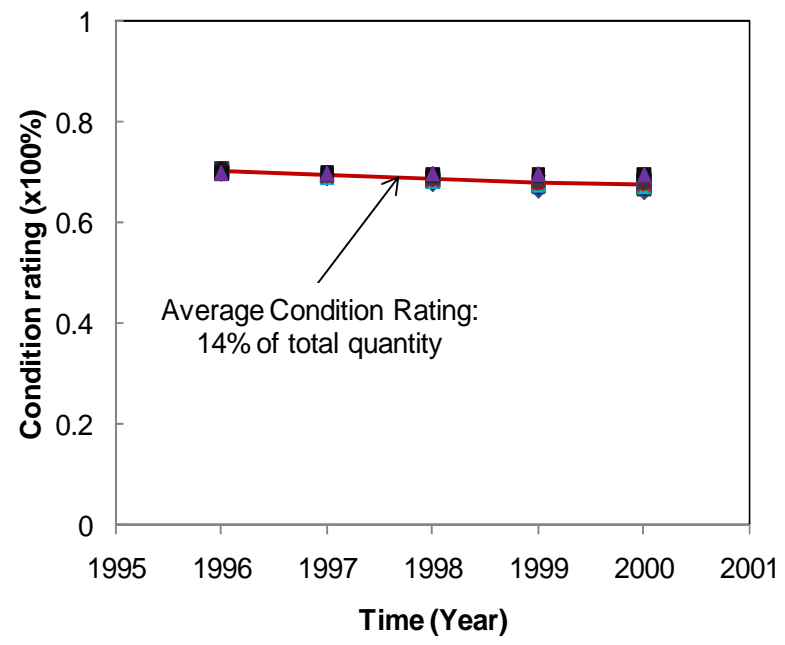

(b) $14 \%$ of total element quantity

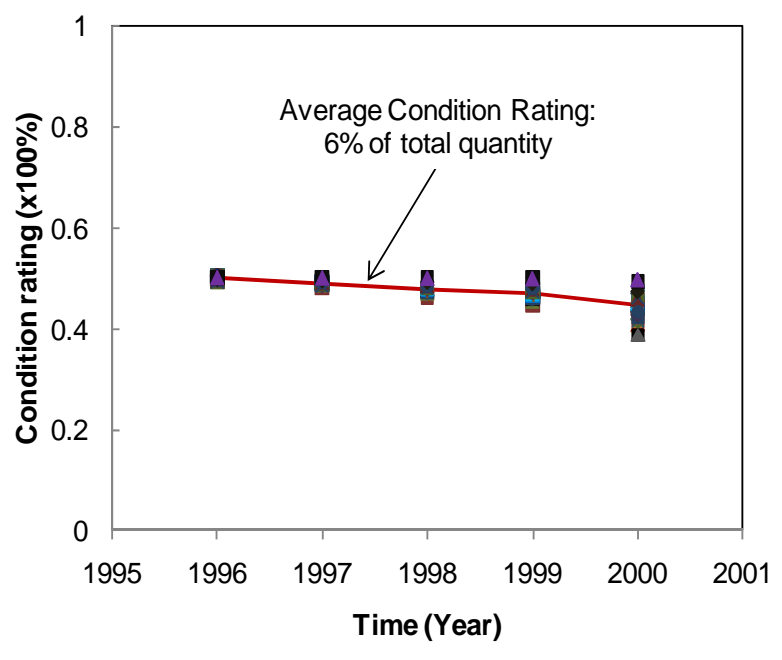

(c) $6 \%$ of total element quantity

Fig. 6. TDNN short-term prediction results for cross-validation (years 1996, 1998, and 2000)

TDNN long-term prediction: Once validated, all historical condition ratings, i.e. assumed condition rating at year of construction completion (year 1966), together with the BPM-generated missing condition ratings (years 1967 to 2000), are used as TDNN inputs. The result of the first one-step-ahead prediction (i.e. year 2001) is added on to the original TDNN input (years 1967 to 2000). Iterations of this one-step-ahead prediction process are continued until year 2010 for a 10year long-term prediction. Figure 7 shows a summary of the predicted condition states for the 10year long-term prediction (years 2001 to 2010) using yearly BPM-generated condition ratings (years 1967 to 2000). The advantage of the proposed deterioration model is that it can provide quantity of each CS thereby overcoming the shortcomings of OCR which ignores critical failure risks in small number of bridge elements with low Condition States (CSs). 


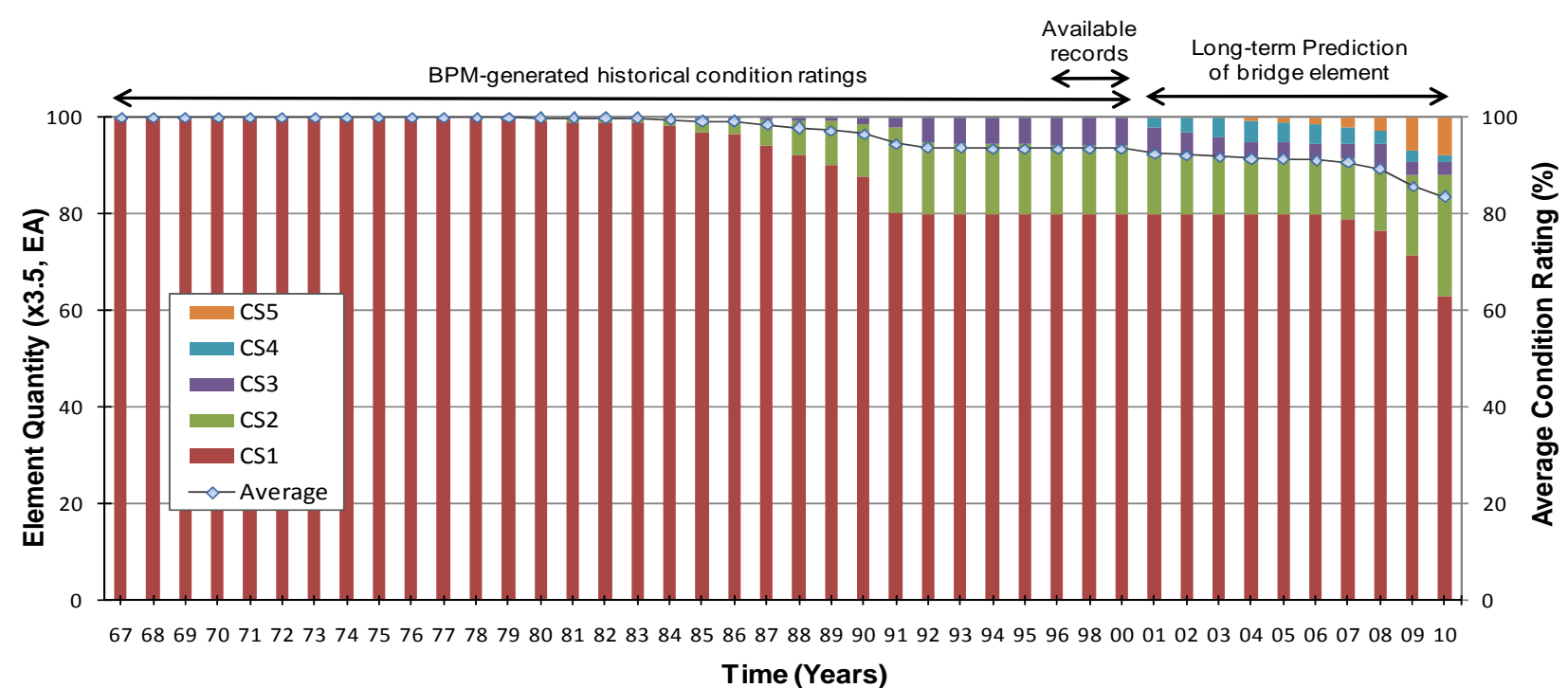

Fig. 7. Summary of predicted condition states

\section{Discussion and Summary}

The previously developed AI-based bridge deterioration modelling technique incorporating the BPM [10] has proven to be able to improve accuracy of long-term prediction. The current model, however, is computationally less efficient due to the time consuming Neural Network training process, which generates a huge amount of data. The BPM-generated condition ratings for each year contain 66 spectra of rating numbers per CS, which is a combination of the learning rates (lr) (i.e. Ir: 0.0 to 0.5 @ 0.1 increment), and momentum coefficients (mc) (mc: 0.0 to 1.0 @ 0.1 increment) in the Neural Network configurations. The number 66 (i.e. 6x11) applies to given element type. The 66 BPM outcomes per CS per year create a huge number of combined scenarios in long-term prediction stage; for four CSs in n years it gives $(66 \times 4) n$. As the initial settings prior to Neural Network training are a result of a random data process, many of the combined scenarios are less meaningful which need to be filtered out to further increase prediction accuracy and efficiency. To achieve this ultimate goal, this study presents a data filtering process as a first step, by which the BPM-generated condition ratings can provide logical information for reliable long-term prediction of bridge elements by TDNN. The TDNN is one of the sensitive and reliable dynamic analysis techniques for signal processes and speech recognitions, because it has a strong ability to detect patterns of dynamic input data. However, irregular noise pattern(s) or illogical pattern(s) leads poor training results.

The first stage of the AI-based deterioration model is to generate unavailable condition ratings. Based on three sets of available condition ratings, the BPM is able to generate 29 missing condition ratings when proper inspection records were missing. The prediction error of the condition rating in year 2000 is $4.2 \%$, which is within the error allowance $( \pm 10 \%)$. The maximum total yearly prediction error is found to be $4.62 \%$ (about 16 out of 350 total elements) in year 2000. This stage also involves data filtering process on all BPM-generated condition rating outcomes. The illogically predicted condition ratings are filtered out which is equivalent to $44 \%$ of the predicted data (i.e. 88 cases out of 198 cases). The resulting maximum and average maximum errors are $3.26 \%$ and $1.49 \%$, respectively. During the second stage, a short-term prediction for cross-validation is performed to re-confirm the prediction accuracy of BPM-generated missing condition ratings and the reliability of TDNN. The results of short-term prediction (years 1996 to 2000) are compared with the existing condition ratings for the same time period (years 1996 to 2000). Some results are over-estimated by $1.25 \%$ in CS3 in year 2000. However, this prediction error is within the error allowance. Therefore, it can be ascertained that all BPM-generated condition ratings can be used as input for TDNN longterm prediction. Upon validation, full historical condition ratings including assumed condition rating at year of construction completion (year 1966), BPM-generated historical condition ratings 
(years 1967 to 1995), and the existing condition ratings (years 1996 to 2000) can be used for TDNN long-term prediction. As seen in Figure 7, the outcomes of TDNN predictions are in the same form as quantitative condition rating inspections. This suggests that the predictions are more descriptive than the typical overall condition rating (OCR) method in terms of elements quantities in each CS.

\section{Conclusion}

The filtering process has helped further improve the prediction accuracy of the AI-based bridge deterioration model. Based on the outcome of the present study, further case studies should be carried out to confirm the methodology developed and to improve time efficiency in typical ANN training process. Further work should also consider the maintenance effects in predicting long-term bridge element performance. Nevertheless, the outcome of the present study is very useful for further development of a reliable bridge deterioration model for BMSs.

\section{Acknowledgement}

The resources used for the present study were provided by the Maryland State Department of Transportation, U.S. The authors wish to thank Messrs Earle Freedman and Matt Zulkowski for providing necessary information requested.

\section{References}

[1] Mauch, M., and Madanat, S. (2001). "Semiparametric Hazard Rate Models of Reinforced Concrete Bridge Deck Deterioration." Journal of Infrastructure Systems, 7(2), 49-57.

[2] Madanat, S., and Ibrahim, W. H. W. (1995). "Poisson Regression Models of Infrastructure Transition Probabilities." Journal of Transportation Engineering, 121(3), 267-272.

[3] Agrawal, A. K., Qian, G., Kawaguchi, A., Lagace, S., Delisle, R., Kelly, B., Weykamp, P., Conway, T. and Dublin, E. (2006). "Deterioration Rates of Typical Bridge Elements in New York." ASCE.

[4] DeStefano D, Grivas A (1998). "Method for estimating transition probability in bridge deterioration models." J Infrastruct Syst, 4(2), 56-62.

[5] Madanat SM, Karlaftis MG, McCarthy PS (1997). "Probabilistic infrastructure deterioration models with panel data." Journal of Infrastructure System, 3(1), 120-125.

[6] Morcous G, Rivard H, Hanna A (2000). "Case-based reasoning system for bridge management." Computing in Civil and Building Engineering, 1363-1370.

[7] Godar B, Vassie R (1999). "Review of existing BMS and definition of inputs for the proposed BMS." Deliverable D4 BRIME Report, PL97-2220.

[8] Lee, J. H., Sanmugarasa, K., Loo, Y. C., and Blumenstein, M. (2008). "Improving the Reliability of a Bridge Management System (BMS) using an ANN-based Backward Prediction Model (BPM)." Journal of Automation in Construction, 17(6), 758-772.

[9] Son, J. B., Lee, J. H., Blumenstein, M., Loo, Y. C., Guan, H., and Panuwatwanich, K. (2009). "Improving reliability of Bridge deterioration model using generated missing condition ratings." 3rd International Conference on Construction Engineering and Management (ICCEM), Jeju, S. Korea, CD-ROM Proceedings.

[10]Son JB, Lee JH, Guan H, Loo YC, Blumenstein M (2010). "ANN-based structural element performance model for reliable bridge asset management." Proc, the 21st Australasian Conf on Mechanics of Structures and Materials, Melbourne, Australia, 775-780. 